\title{
PROGRESS TOWARDS A LOCAL EXPRESSION FOR RADIATION REACTION
}

\author{
RACHEL ANNE CAPON \\ Max-Planck-Institut für Gravitationsphysik, Albert-Einstein-Institut \\ Schlaatzweg 1, D-14473 Potsdam, Germany* \\ E-mail: rachel@aei-potsdam.mpg.de
}

\begin{abstract}
We report on progress towards finding a local expression for radiation reaction for a particle orbiting a Kerr black hole. The Dirac-Gal'tsov approach is described. For the case of a scalar particle in a circular orbit of a Schwarzschild black hole, an explicit calculation is done via this method and shown to be in agreement with overall energy conservation. A possible approach to the case of more general orbits is also discussed.
\end{abstract}

1. A local approach to the two body problem. The two-body problem in General Relativity is of both theoretical and experimental significance, yet it is intractable and the solution will require either numerical relativity or some kind of analytic approximation. For the latter there are two possibilities: firstly post-Newtonian techniques - the main focus of this part of the meeting - which are valid provided the velocity, $v \ll 1$; secondly, the restricted mass approximation $\mu \ll M$, which is valid for any $v$. Poisson [1] was the first to use this second approximation in conjunction with a slow-motion approximation. By this method post-Newtonian expansions of the luminosity have been obtained to a much higher order than previously achieved [2, 3, 4].

In our work we consider the restricted mass case, in particular a mass $\mu$ orbiting a black hole of mass $M \gg \mu$. The motion of $\mu$ will be approximately a geodesic of the black hole. The self-field of $\mu$ will then induce corrections of order $\frac{\mu}{M}$, which give rise to non-geodesic motion of various kinds, notably radiation reaction, which we associate with the lowest order non-geodesic motion. At this order, the self-field, $\psi$, of $\mu$ is described by a perturbation equation on the background space time of the black hole of the form,

$$
\square \psi=4 \pi \sqrt{-g} T,
$$

with source term $T$ due to $\mu$.

1991 Mathematics Subject Classification: 83C30, 83C35.

* On leave from Dept. Physics and Astronomy, U.W.C.C., Cardiff, Wales.

The paper is in final form and no version of it will be published elsewhere. 
There are two ways in which the radiation reaction could be calculated. The first possibility is using energy and angular-momentum balance, i.e. for a geodesic, $\left\{\frac{d E}{d t}, \frac{d J}{d t}\right\}$ are calculated at future null infinity and then subtracted from the orbit at the appropriate retarded time. However, for this to be valid, a slowly changing orbit and stationary field of $M$ must be assumed. Furthermore the orbit must be fully characterised by $E$ and $J$. This is not always the case - to characterise non-equatorial orbits around a Kerr black hole the Carter constant is also necessary, but cannot be incorporated into such a balance argument.

The other possibility is to look for a local method, i.e. to calculate the self-field of $\mu$ to linear order and let it react on $\mu$. Our aim is thus to find a local expression for the radiation reaction force. Unlike post-Newtonian calculations this will be valid also in the strong-field case and for any velocity of $\mu$, not just slow-motion. It will also be applicable to all orbits of a Kerr black hole, including those for which the global energy balance method fails.

The chief difficulty in calculating such a local radiation reaction force at $\mu$ is that the retarded Green's function and thus the self-field become singular at the particle. We propose to overcome this problem by adopting the Dirac-Gal'tsov approach. Namely, the retarded Green's function can be written as the sum of two terms,

$$
G^{\mathrm{ret}}\left(x, x^{\prime}\right)=\frac{1}{2}\left[G^{\mathrm{ret}}\left(x, x^{\prime}\right)+G^{\mathrm{adv}}\left(x, x^{\prime}\right)\right]+\frac{1}{2}\left[G^{\mathrm{ret}}\left(x, x^{\prime}\right)-G^{\mathrm{adv}}\left(x, x^{\prime}\right)\right] .
$$

The first of these terms is symmetric in time. It therefore contains no radiation but is responsible for the Coulomb-type divergences on the particle. The second term is antisymmetric in time and contains the radiation. Dirac [5] showed that, for the classical electron, this term is non-divergent. The scheme is then to use only this radiative Green's function,

$$
G^{\mathrm{rad}}\left(x, x^{\prime}\right)=\frac{1}{2} G^{\mathrm{ret}}\left(x, x^{\prime}\right)-\frac{1}{2} G^{\mathrm{adv}}\left(x, x^{\prime}\right),
$$

to obtain the radiation field,

$$
\psi^{\mathrm{rad}}(x)=\int G^{\mathrm{rad}}\left(x, x^{\prime}\right) T\left(x^{\prime}\right) \sqrt{-g\left(x^{\prime}\right)} d^{4} x^{\prime} .
$$

Here the integration is over the whole future and past history of the particle. We would like to know how many particle orbits are significant for the radiation reaction - this question may only be addressed by using a local method.

2. Gal'tsov's formalism. Gal'tsov [6] developed a formalism to compute $G^{\text {rad }}\left(x, x^{\prime}\right)$ in general relativity for scalar, electromagnetic and gravitational perturbations of a Kerr black hole based on Chrzanowski and Misner's [7, 8] factorised form of the Green's functions. For simplicity we first consider scalar perturbations of a Schwarzschild black hole. This should yield valuable qualitative information and the method can be generalised to the other cases. The Green's function is built from solutions of the homogeneous perturbation equation, $\square \psi=0$. Separating the variables we obtain

$$
\psi(x)=\sum_{l=0}^{\infty} \sum_{m=-l}^{l} \int d \omega \frac{u(r)}{r} S_{l m}(\theta) e^{i m \phi} e^{-i \omega t},
$$


where $u(r)$ satisfies the Regge-Wheeler equation

$$
\frac{d^{2} u}{d r_{*}^{2}}+\left[\omega^{2}-V(r)\right] u(r)=0,
$$

with $r_{*}=r+2 M \log (r / 2 M-1)$ and

$$
V(r)=\left(1-\frac{2 M}{r}\right)\left(\frac{l(l+1)}{r^{2}}+\frac{2 M}{r^{3}}\right) .
$$

Two linearly independent solutions for $u$ can be characterised in the following way,

$$
\begin{aligned}
& u^{\text {in }} \rightarrow \begin{cases}A_{\text {out }}(\omega) e^{i \omega r_{*}}+A_{\text {in }}(\omega) e^{-i \omega r_{*}}, & \text { as } r_{*} \rightarrow \infty, \\
e^{-i \omega r_{*}}, & \text { as } r_{*} \rightarrow-\infty,\end{cases} \\
& u^{\text {up }} \rightarrow \begin{cases}e^{i \omega r_{*}}, & \text { as } r_{*} \rightarrow \infty, \\
A_{\text {in }}(\omega) e^{i \omega r_{*}}+\bar{A}_{\text {out }}(\omega) e^{-i \omega r_{*},}, & \text { as } r_{*} \rightarrow-\infty .\end{cases}
\end{aligned}
$$

We also use the complex conjugate solutions $u^{\text {out }}=\bar{u}^{\text {in }}$ and $u^{\text {down }}=\bar{u}^{\text {up }}$. Then

$$
\begin{aligned}
G^{\mathrm{rad}}\left(x, x^{\prime}\right)=\sum_{l, m} \int d \omega\left(\frac{-1}{4 \pi i \omega}\right) \frac{1}{A_{\text {in }} \bar{A}_{\text {in }}} e^{-i \omega\left(t-t^{\prime}\right)} S_{l m}(\theta) \bar{S}_{l m}\left(\theta^{\prime}\right) e^{i m\left(\phi-\phi^{\prime}\right)} \\
\times\left\{\frac{u^{\text {out }}(r)}{r} \frac{\bar{u}^{\text {out }}\left(r^{\prime}\right)}{r^{\prime}}+\frac{u^{\text {down }}(r)}{r} \frac{\bar{u}^{\text {down }}\left(r^{\prime}\right)}{r^{\prime}}\right\}
\end{aligned}
$$

We may use this in (4) above, together with the source term,

$$
T(x)=q \int \delta^{(4)}(x, z(\tau))(-g)^{-\frac{1}{2}} d \tau
$$

assumed here to be given by a delta-function. The particle has world-line $z(\tau)$ and scalar charge $q$. The radiative force is then given by

$$
f_{\mu}^{\mathrm{rad}}=q \frac{\partial \psi^{\mathrm{rad}}}{\partial x^{\mu}} .
$$

The validity of such a method in a curved space-time is open to question. Nevertheless we have chosen to investigate it in the hope that it will yield further insight into the nature of the radiation reaction force.

3. Particle in a circular orbit. To lowest order the path of $\mu$ can be taken as a geodesic. As the simplest case we therefore consider $\mu$ to be on a circular orbit $\left(r=r_{0}, \phi=\right.$ $\Omega t)$ around $M$ and to cause a scalar perturbation field to propagate on the background black hole space-time. Then, implementing Gal'tsov's $G^{\text {rad }}(10)$, we explicitly calculate the $t$-component of the local radiation reaction force,

$$
\begin{aligned}
f_{0}^{r a d}=\frac{q}{2 u_{z}^{0}} \sum_{l, m} \frac{1}{A_{\text {in }} \bar{A}_{\text {in }}} e^{-i m \Omega t} & S_{l m}(\theta) e^{i m \phi} \bar{S}_{l m}\left(\frac{\pi}{2}\right) \times \\
& \times\left.\left\{\frac{u^{\text {out }}(r)}{r} \frac{\bar{u}^{\text {out }}\left(r_{0}\right)}{r_{0}}+\frac{u^{\text {down }}(r)}{r} \frac{\bar{u}^{\text {down }}\left(r_{0}\right)}{r_{0}}\right\}\right|_{\omega=m \Omega}
\end{aligned}
$$

and the local energy loss averaged over one orbit,

$$
E_{\text {ave }}=\left.\frac{q}{2 \Omega\left(u_{z}^{0}\right)^{2}} \sum_{l, m} \frac{1}{A_{\text {in }} \bar{A}_{\text {in }}}\left|S_{l m}\left(\frac{\pi}{2}\right)\right|^{2}\left\{\frac{\left|u^{\text {out }}\left(r_{0}\right)\right|^{2}}{r_{0}^{2}}+\frac{\left|u^{\text {down }}\left(r_{0}\right)\right|^{2}}{r_{0}^{2}}\right\}\right|_{\omega=m \Omega} .
$$


Here $u_{z}^{0}$ is the zero-component of the particle 4-velocity.

We compared this to the energy fluxes, averaged over one period, at the horizon and infinity, obtained using only the retarded Green's function. For this case we were thus able to show overall energy conservation, namely that for our local expression for the energy flux (14) above, the second term corresponds to energy lost down the event horizon to the black hole and the first term to energy which propagates out to infinity.

4. Contributions for a general orbit. We also wish to compute the frequency integral in (10) for more general orbits. Frequencies for which $A_{\text {in }}(\omega)=0$ correspond to the quasi-normal modes of the black hole. The integrand of $G^{\mathrm{rad}}$ will have singularities at these (complex) frequencies. (This is not apparent in the normalization of $u^{\text {in }}$ and $u^{\text {up }}$ chosen by Gal'tsov.) To see explicitly the effect of the quasi-normal modes we need to extend the inverse Fourier Transform above into the complex frequency plane. In order to do this consistently it then becomes necessary to consider the retarded and advanced Green's functions separately.

For the retarded Green's function the inverse Fourier Transform will be given by

$$
\begin{aligned}
G^{\mathrm{ret}}\left(x, x^{\prime}\right)=\sum_{l, m} \int_{-\infty+i c}^{+\infty+i c} d \omega\left(\frac{-1}{4 \pi i \omega A_{\text {in }}(\omega)}\right) e^{-i \omega\left(t-t^{\prime}\right)} S_{l m}(\theta) \bar{S}_{l m}\left(\theta^{\prime}\right) e^{i m\left(\phi-\phi^{\prime}\right)} \times \\
\times\left\{\frac{u^{\mathrm{in}}(r)}{r} \frac{u^{\mathrm{up}}\left(r^{\prime}\right)}{r^{\prime}} \theta\left(r^{\prime}-r\right)+\frac{u^{\mathrm{up}}(r)}{r} \frac{u^{\mathrm{in}}\left(r^{\prime}\right)}{r^{\prime}} \theta\left(r-r^{\prime}\right)\right\},
\end{aligned}
$$

where $c$ is a real constant greater than zero. When this contour is closed in the lower half of the complex $\omega$ plane (as shown in fig. 1) there will be contributions from the quasi-normal mode poles, $A_{\text {in }}(\omega)=0$, from the branch cut ( $\omega=0$ is a branch point) and from the high-frequency arcs $(|\omega| \rightarrow \infty)$.
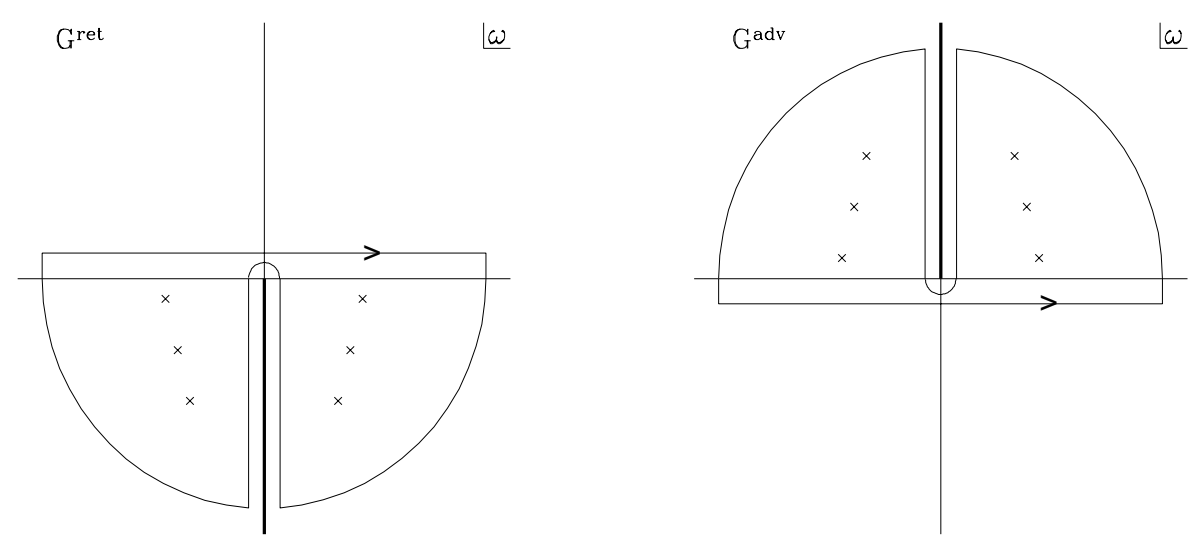

Fig. 1. Continuation of frequency integral for $G^{\text {ret }}$ and $G^{\text {adv }}$ into the complex plane. The branch cut is indicated in each case by a thick line and quasi-normal mode frequencies by crosses.

The situation for $G^{\text {adv }}$ is similar but there the poles, due to the time-reversed quasinormal modes, $\bar{A}_{\text {in }}(\omega)=0$, and the branch cut are in the upper half of the $\omega$ plane. 
Each of these contributions will need to be considered for both the retarded and advanced Green's function and then compared to see where any cancellation occurs when the radiative Green's function is taken.

For the retarded Green's function, for times

$$
t-t^{\prime}<\left|\xi-\xi^{\prime}\right|
$$

the high-frequency arc vanishes when closed in the upper half plane. $G^{\text {ret }}=0$ as expected for such acausal times. The high-frequency arcs in the lower half plane can be shown to vanish for times such that

$$
t-t^{\prime}>\xi+\xi^{\prime}
$$

Here we have defined a new radial-type coordinate, $\xi$, by

$$
\xi=\sqrt{r^{2}-4 M^{2}}-4 M \log \left[\sqrt{\frac{r}{2 M}+1}-\sqrt{\frac{r}{2 M}-1}\right] .
$$

Note that $\xi \sim r_{*}$ as $r \rightarrow \infty$

5. Summary and discussion of future work. Our aim is to find a local expression for gravitational radiation reaction. We have approached the problem by employing the restricted mass approximation and the Dirac-Gal'tsov method. Unlike post-Newtonian calculations, our results will be applicable also to strong field situations, high particle velocities and general orbits of a Kerr black hole.

We have computed the local energy loss due to radiation reaction for a scalar particle in a circular orbit around a Schwarzschild black hole by the Dirac-Gal'tsov method. Furthermore, in this case we have demonstrated overall energy conservation by comparing the local result with the energy fluxes at infinity and at the horizon.

For more general orbits we proposed considering the retarded and advanced Green's functions separately, which will enable us to continue the inverse Fourier Transform integral into the complex frequency plane. We then expect contributions from the quasinormal modes, the branch cut and the high frequency arcs. For the retarded Green's function the latter have been shown to give vanishing contribution for acausal times, $t-t^{\prime}<\left|\xi-\xi^{\prime}\right|$ and "late" times, $t-t^{\prime}>\xi+\xi^{\prime}$. To calculate the Green's function for other "early" times it may be necessary to first sum over the spherical harmonics. The quasi-normal mode and branch cut contributions have still to be calculated specifically for this problem, although they have already been considered by several authors (see e.g. $[9,10])$.

In these calculations we should not lose sight of our original goal, namely to calculate the radiative Green's function and thus find a local expression for radiation reaction and also the extent to which the particle history is significant for the radiation reaction. These calculations are first envisaged to be carried out in the simplified case of a scalar particle orbiting a Schwarzschild black hole. We then wish to investigate the generalization of the calculation to gravitational waves and to the Kerr black hole.

Acknowledgements. I would like to thank my supervisor, Bernard F. Schutz, for suggesting the problem and for many helpful discussions. I would also like to thank 
Nils Andersson for all his help and for proposing the high frequency solution to the Regge-Wheeler equation which I used to carry out the integral over the high frequency arcs.

\section{References}

[1] E. Poisson, Gravitational radiation from a particle in circular orbit around a black hole. i. analytical results for the nonrotating case. Phys. Rev. D, 47:1497-1510, 1993.

[2] H. Tagoshi and M. Sasaki, Post-Newtonian expansion of gravitational waves from a particle in circular orbit around a Schwarzschild black hole. Prog. Theor. Phys., 92:745, 1994.

[3] E. Poisson and M. Sasaki, Gravitational radiation from a particle in circular orbit around a black hole. v. black-hole absorption and tail corrections. Phys. Rev. D, 51:5753, 1995.

[4] H. Tagoshi, M. Shibata, T. Tanaka, and M. Sasaki, Post-Newtonian expansion of gravitational waves from a particle in circular orbits around a rotating black hole: Up to $O\left(v^{8}\right)$ beyond the quadrupole formula. Phys. Rev. D, 54:1439, 1996.

[5] P. A. M. Dirac, Classical theory of radiating electrons. Proc. Roy. Soc. Lond. A., 167:148-169, 1938.

[6] D. V. Gal'tsov, Radiation reaction in the Kerr gravitational field. J. Phys. A, 15:37373749, 1982.

[7] P. L. Chrzanowski and C. W. Misner, Geodesic synchrotron radiation in the Kerr geometry by the method of asymptotically factorized Green's functions. Phys. Rev. D, 10:1701-1721, 1974.

[8] P. L. Chrzanowski, Vector potential and metric perturbations of a rotating black hole. Phys. Rev. D, 11:2042-2062, 1975.

[9] N. Andersson, Excitation of Schwarzschild black-hole quasinormal modes. Phys. Rev. D, 51:353-363, 1995.

[10] E. S. C. Ching, P. T. Leung, W. M. Suen, and K. Young, Wave propagation in gravitational systems: Late time behaviour. Phys. Rev. D, 52:2118-2132, 1995. 